\title{
R Script for Classifying Patterns of Urban Growth
}

\author{
Joan Perez ${ }^{1 \text { [0000-0003-3003-0895], Alexandre Ornon }}{ }^{\text {[0000-0001-9806-1319] }}$ and Hiroyuki \\ Usui ${ }^{2[0000-0002-2447-5372] ~}$ \\ ${ }^{1}$ Université Côte-Azur, CNRS, ESPACE, Nice, France \\ 2 The University of Tokyo, Department of Urban Engineering, Housing and Urban Analysis \\ laboratory, Tokyo, Japan \\ \{joan.perez, alexandre.ornon\}@univ-cotedazur.fr \\ usui@ua.t.u-tokyo.ac.jp
}

\begin{abstract}
This paper presents a script that classify spatial patterns of residential urban growth using a morpho-structural approach. The script performs a combination of variography analysis and morphological closings over buildings possessing a residential function in 2002 and 2017 within a region located in southern France named Centre-Var. The different bounding regions then allow classifying new residential buildings into different categories according to their degrees of clustering/scattering and to their locations regarding existing urban areas. Preliminary results show that this protocol is able to provide useful insights regarding the degree of contribution of each new residential building to different patterns of urban growth (clustered infill, scattered infill, clustered edgeexpansion, scattered edge-expansion, clustered leapfrog and scattered leapfrog). Open-access to the script and to the test region data is provided.
\end{abstract}

Keywords: Classification, Morphology, Geoprocessing, Variography, Geostatistics.

\section{Introduction}

A growing number of sources, from intergovernmental organizations to academics [1, $2,3]$ are increasingly directing the attention on the urgency of transforming the way urban spaces are built and managed. Amongst these sources, the main identified issue is often sprawl which is deemed has a non-sustainable dynamic regarding environmental issues $[2,3,4]$. Yet, the literature on urban sprawl and its different patterns is ambiguous, or even confusing since no consensus is emerging in the academics $[5,6,7]$. This is without mentioning other patterns of growth which are not directly related to sprawl, such as redevelopment of existing urban areas, urban scattering, leapfrog urbanization, etc. Numerous denominations of somewhat similar patterns also exist in the literature such as central-city revival and urban regeneration for urban redevelopment [8]; edge expansion for continuous sprawl [9], etc. Even if sprawl remain the main scapegoat, other patterns of urban development are also pointed out as hardly sustainable [10]. As suggested by Liu et al., [9], urban growth spatially operates following three main possibilities: infill, edge-expansion and leapfrog. Solely 
focusing on sprawl, Galster et al., [6] proposes a conceptual definition based on eight dimensions. Among them, one is of particular interest in this research: clustering. It is defined as the degree to which development is tightly bunched together. Yet, the degree of clustered development appears not only relevant for edge-expansion patterns, but also for infill and leapfrog. Based on these observations, it could be possible to classify new residential buildings into the aforementioned patterns of urban growth according to both location and clustered measures of existing and new buildings. Since certain patterns are deemed more sustainable than others, such as compact or resilient models of development $[10,11]$, such a classification could provide useful insights regarding into which pattern new building falls.

The goal of this paper is to present an R script that classify spatiotemporal patterns of residential urban growth within a region located in southern France named CentreVar. The protocol can be described as a location-based morpho-structural approach which combines variography analysis and morphometry (mathematical morphology analysis applied to vector objects). It first detects thresholds of residential building agglomerations in 2002 and 2017 and then performs several morphological closings according to building locations. The bounding regions obtained through the morphological closings then allow classifying the new residential buildings into six different spatial patterns of urban growth. The script makes use of easily accessible data: GIS layers of residential building footprints. In order to contribute to the open science movement, open-access to the source code and to the test region data is provided.

This paper is organized as follows. Section 2 introduces the test region and the primary data. Section 3 presents and details each step of the script. Section 4 presents the preliminary results obtained on the test region. Section $\mathbf{5}$ concludes the paper with a discussion on future applications and developments of the protocol.

\section{Test Region and Data Presentation}

The region in which the method is applied is the center of the Var department in southern France. The extent of the case study goes from the cities of Brignoles to Le Muy and also includes Draguignan, Vidauban and Le Luc. This area, named CentreVar, is located close to three major cities: Marseille, Toulon and Nice. Due to the proximity of these metropolitan areas, this region is sustaining fast processes of urbanization (increase of population, sprawl, urban redevelopment, etc.). According to INSEE $^{1}$, Centre-Var population in 1999 was 157.919 inhabitants and 210.071 in 2016, thus showing an impressive growth of $33.02 \%$.

\footnotetext{
${ }^{1}$ French national institute for statistical and economic studies.
} 


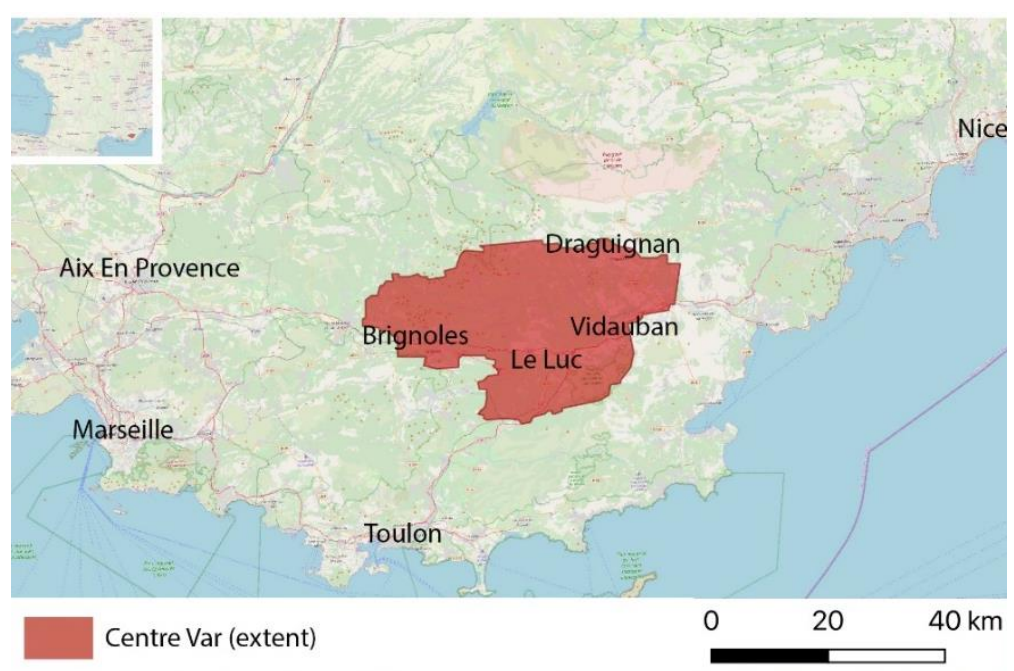

Fig. 1. Centre-Var Presentation. Background: OpenStreetMap, 2020.

The BD TOPO® datasets from the French National Geographic Institute (IGN) are extracted and filtered according to the Centre-Var boundaries. BD TOPO® are GIS layers of building footprints (polygons that represent building shapes on a twodimensional plane) where buildings are digitized as single-part polygons. Two dates are retained: 2002 and 2017. Buildings not possessing any residential functions are filtered out of the datasets using the specialization attribute included in the primary data. Since these data includes small polygons usually associated to residential buildings (garage, pergola, sheds, etc.), light buildings and small structures below $20 \mathrm{~m}^{2}$ are filtered out. Data are finally harmonized into a single point feature class (centroids of polygons) possessing two attribute columns indicative of the building presence for each period: pres2002 and pres2017 (modalities: "1" for building presence; "0" otherwise). The prepared dataset is ultimately made of 82.249 centroids of residential buildings in 2017, from which 63.238 were also present in 2002. Data are compiled into a GeoPackage file named CV_PT_Ext_0217 containing two layers: the centroids, named CV_Building_PT and the boundaries of Centre-Var, named Centre_Var_extent.

\section{$3 \quad$ R Script Presentation and Application}

The script starts with a subsection that provides information on the $\mathrm{R}$ session and packages versions. Necessary packages are then loaded. A second subsection reads the GeoPackage and subsequently divides the points into three simple feature geometry objects: the centroids present in 2002 Pt_2002.sp, those present in 2017 Pt_2017.sp and the new centroids that appeared between 2002 and 2017 Pt_2017_new.sp. The script is then organized in three parts, as follows: 
Part one of the script creates a RasterLayer object of 50 meters of resolution within the case study boundaries using the raster function from the "raster" package. cellFromXY counts the number of points per raster cell for 2002 and 2017. A crossvariogram is then calculated for these two variables using the variogram function of the "gstat" package. Variography allows exploring the spatial structure inherent to the point distributions. The small variation of auto-correlation explains a local change in the way that buildings are structured. This variation is considered as a micro-structure and sets the threshold of the agglomeration distance within the point distribution. As displayed in Fig. 2, the first micro-structure threshold is identified at 227 meters, which is rounded according to the grid resolution at 250 meters.

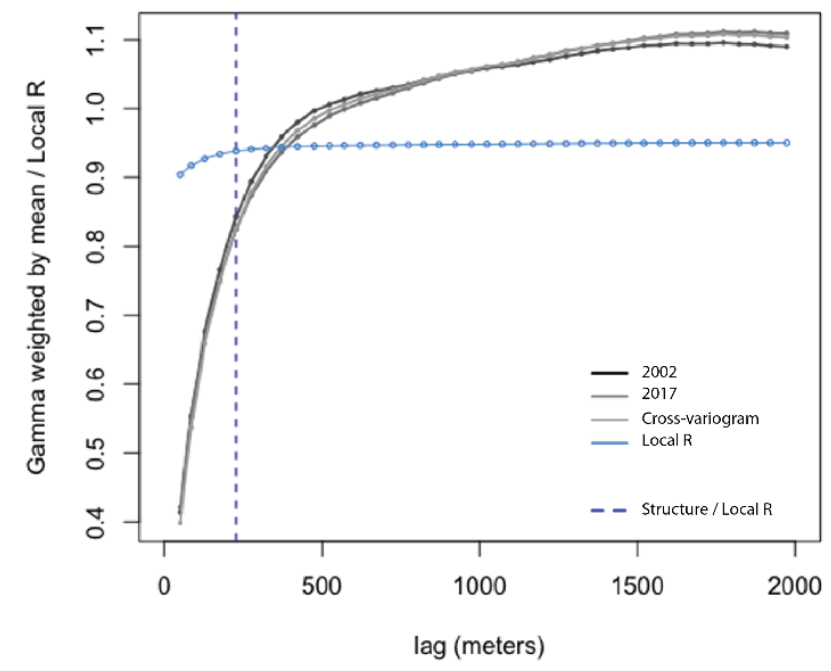

Fig. 2. Cross-Variogram for 2002 and 2017 Residential Buildings (50 meters building densities)

Offsets around the 2002 and 2017 point features are then created in order to extract built-up areas using the buffer function of the "raster" package. These operations, named morphological closings [12], are composed of a dilatation followed by an erosion algorithm. They allow linking close by centroids, ignoring small holes and interstices. The micro-structure threshold of 250 meters detected by the analysis of the cross-variogram sets the buffering distance from each point (radius $r=250 / 2$ ). For both closings, a filter is applied in order to remove the small isolated surfaces artificially generated by the dilation algorithm, which are the surfaces below the lower limit of $\pi r^{2}$. The last section of part one performs the difference between the closings of 2017 and 2002 and the difference between the extent of the case study and the closing of 2017 using the gDifference function from the package "rgeos". 
Part two of the script divides the new centroids Pt_2017_new.sp into three subsets through basic operations of intersect using the st_intersection function of the "sf" package. Point_evo_inside_2002 are the centroids within the 2002 morphological closing, Point_evo_outside 2017 are the centroids outside the 2017 closing and Point_evo_diff_0217 are the centroids located both outside 2002 and inside 2017 closings. A downscaling is performed through the creation of a RasterLayer object of 25 meters of resolution. The number of new building centroids is then counted, using once again raster and cellFromXY functions. Performing a downscaling is relevant since, at this stage, the focus of the study is no more on the inclusion/exclusion of new buildings within existing urban areas but rather on their clustered/scattered properties. Since each subset is representative of a peculiar trajectory, regular variograms are calculated within each subset (instead of a cross-variogram). As displayed in Fig. 3, they yield thresholds of 113 meters for Point_evo_inside_2002 (rounded at 125 meters), 161 meters for Point_evo_diff_ 0217 (rounded at 150 meters), and 238 meters for Point_evo_outside_2017 (rounded at 225 meters). Morphological closings are once again performed for each subset using the different identified thresholds as buffering radiuses.

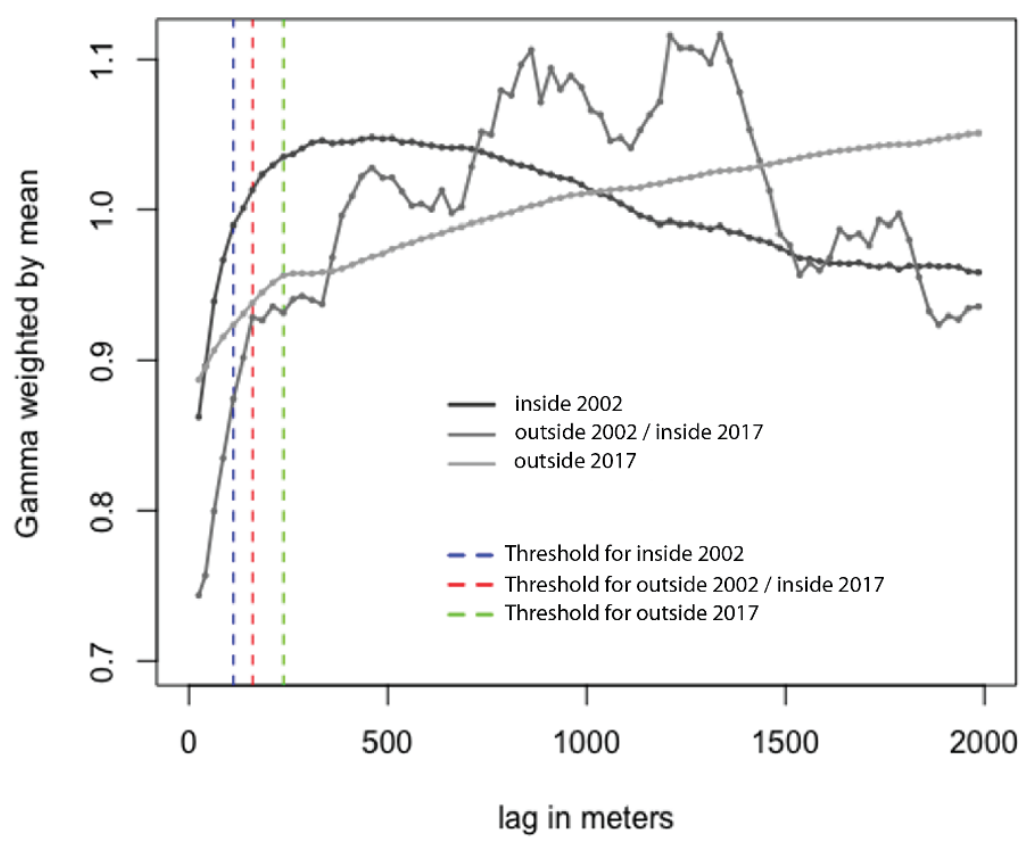

Fig. 3. Variograms for Point_evo_inside_2002, Point_evo_outside_2017 and Point_evo_diff_0217 (25 meters building densities)

Part three performs the final classification by selecting the building centroids encroaching upon the different buffers, ultimately yielding the following categories: 
clustered infill, scattered infill, clustered edge-expansion, scattered edge-expansion, clustered leapfrog and scattered leapfrog. The different geometries are also clipped together once again using the function gDifference. Several plots and maps are displayed and results are recorded, including the variogram samples. Result layers are added within the original GeoPackage file.

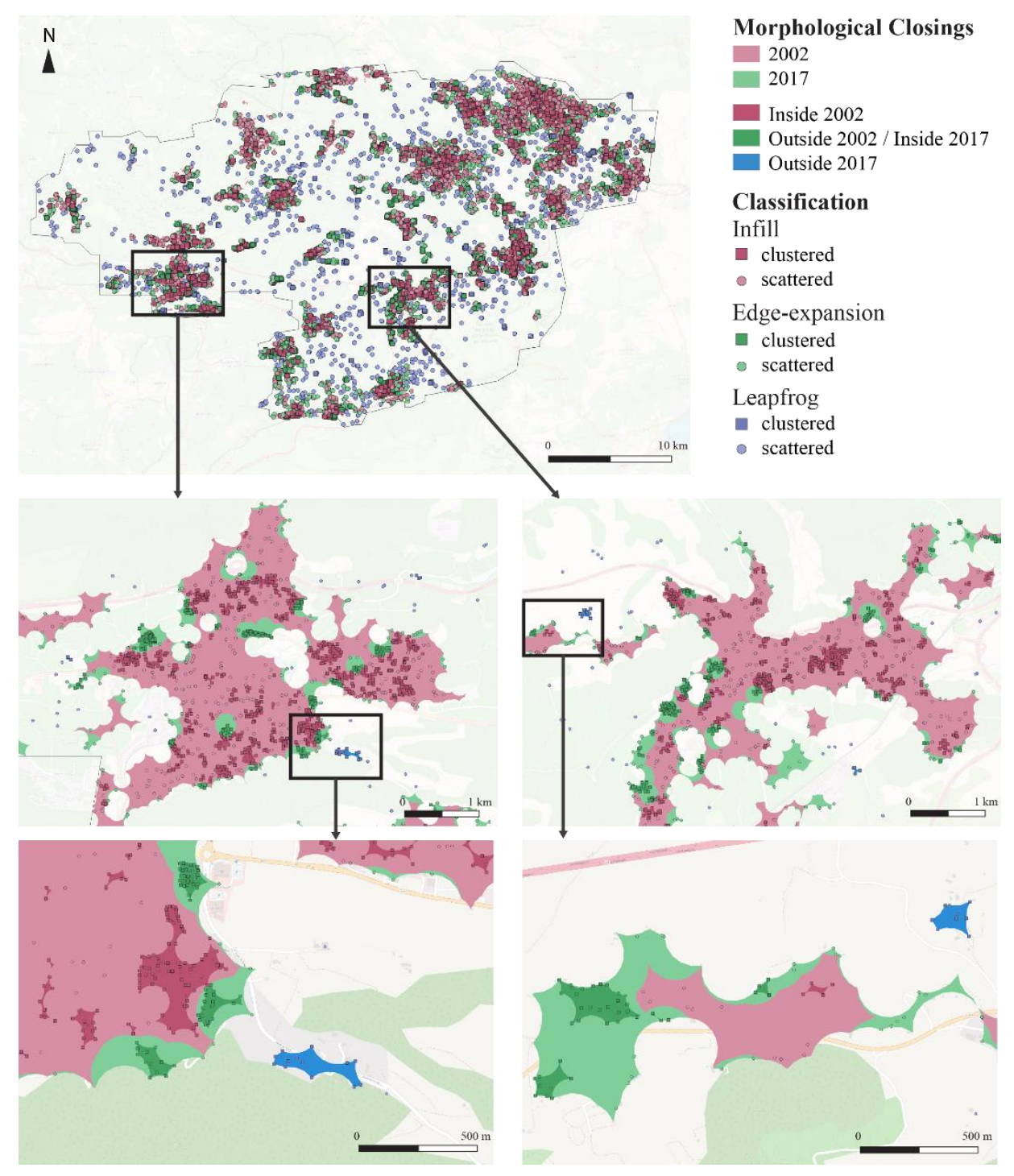

Fig. 4. Mophological Closings and Classification Results. Focus on Brignoles (left) and Le Luc (right). Background: OpenStreetMap, 2020. 
As Fig.4 shows, the three categories (infill, edge-expansion and leapfrog), as well as their subdivisions (clustered and scattered), clearly stand out. Both Brignoles and Le Luc are mostly concerned by infill and edge-expansion patterns. They are both gaining a lot of ground on non-urban spaces from existing urban structures, whereas leapfrogs exist but remain limited comparing to the other patterns. The script, as well as the data related to the Centre-Var test region have been made available (supplementary materials 1).

\section{$4 \quad$ Preliminary Results}

New residential buildings have been successfully classified into three categories: infill, edge-expansion and leapfrog; each of the latter further subdivided into clustered and scattered patterns.

$66.71 \%$ of the new buildings are located within urban structures that were already present in 2002. These buildings are infill patterns of urban growth directly contributing to urban densification. Among this group, the clustered pattern corresponds to the infilling of urban vacuities at the scale of urban blocks or strips of land that were previously not constructed, or at least not fully occupied by residential buildings. This category is also corresponding to various projects of urban redevelopment and regeneration of old urban fabrics. The scattered pattern can however be described as additions of adjacent buildings or infillings of urban vacuities at the scale of the plot, within existing urban blocks. It can be safely assumed that these new buildings are not planned nor coordinated by administrative authorities and promoters, but are rather the outcome of individual initiatives.

$24.85 \%$ of the new buildings, classified as edge-expansion patterns, are directly responsive for urban sprawl. They are the ones gaining ground on non-urban spaces from existing urban structures, thus prolonging the latter in an unbroken fashion. The clustered ones are corresponding to compact new peripheral neighborhoods. Scattered urban development are also contributing to urban expansion. Yet, as compared to the former, this pattern is not compact, thus contributing to low-density urban expansion.

$8.44 \%$ of the new building centroids are located outside of the 2017 morphological closing, which means that these new buildings are not contributing to urban growth in the sense of continuity. The clustered pattern be described as compact leapfrog urban developments. These patterns are widespread in numerous countries but, as highlighted in Fig. 4, it appears that it is not so much the case in Centre-Var. This phenomenon is often described in the academic literature as compact but yet not continuous urban development $[13,14]$. In Brignoles and Le Luc only two small pockets of such emerge. The scattered leapfrog pattern can, for its part, be described as low-density urban development. As compared to the clustered pattern, the hypothesis is that most of these new buildings are single family homes surrounded by large private gardens and villas of complex shapes. 


\section{$5 \quad$ Discussion and Future Developments}

The script presented in this paper allows classifying new residential buildings into six different categories though a combination of variography and morphometry. It yields the following categories: infill, edge-expansion and leapfrog; each of the latter further subdivided into clustered and scattered patterns. As shown in the application to all new residential buildings between 2002 and 2017 in the Centre-Var region, this protocol is able to provide useful insights regarding into which model of development the new residential buildings fall. As highlighted in the literature, some models of development are more sustainable than others. On that basis, 4.726 residential buildings ( $24.85 \%$ of the distribution) are directly responsive for sprawl in the sense of continuity while 1.603 buildings can be described as leapfrog patterns of development (8.44\%). The rest of the distribution are characterized by infill densification patterns. Both clustered and scattered patterns are increasing the density of existing urban spaces and are thus in line with compact city theories. Yet, a fieldwork step is required to validate these preliminary results. The script also has to be tested in other areas, notably within more urbanized regions such as metropolitan areas.

The script will be improved in several directions. First, alternatives will be sought for some functions that are time-consuming regarding computation, such as variogram and gDifference. Second, the script will be simplified to make it reproducible to any area. Since it only requires GIS layers of residential building footprints, it could evolve into a decision tool for the evaluation and quantification of spatial patterns of urban growth.

\section{References}

1. United Nations.: Transforming our World: The 2030 Agenda for Sustainable Development. United Nations, New York, (2015).

2. European Environment Agency. Urban sprawl in Europe, The ignored challenge. European Commission report, 60 p., (2006).

3. Verderber, S.: Sprawling Cities and Our Endangered Public Health. Routledge, London, United Kingdom, (2012).

4. Brueckner, JK.: Urban Sprawl: Diagnosis and Remedies. International Regional Science Review, 23(2), pp. 160-171, (2000).

5. Bhatta, B., Saraswati, S. and Bandyopadhyay, D. Urban sprawl measurement from remote sensing data. Applied Geography, 30(4), pp. 731-740, (2010).

6. Galster, G., Hanson, R., Ratcliffe, M., Wolman, H., Coleman, S. and Freihage, J.: Wrestling Sprawl to the Ground: Defining and Measuring an Elusive Concept. Housing Policy Debate, 12(4), pp. 681-717, (2001).

7. Bhatta, B.: Analysis of Urban Growth and Sprawl from Remote Sensing Data. Advances in Geographic Information Science, Springer, 172 p, (2010).

8. Smith, N. The New Urban Frontier, Gentrification and the Revanchist City. New York: Routledge, (1996).

9. Liu, Z.; He, C.; Wu, J. General Spatiotemporal Patterns of Urbanization: An Examination of 16 World Cities. Sustainability, 8, 41, (2016). 
10. Jenks, M., Burton, E. and Williams, K.: The Compact City: A Sustainable Urban Form?, Spon Press, 360 p, (1996).

11. Burdett, R. et Rode, P.: Shaping Cities in an Urban Age. Phaidon Press, 448 p, (2018).

12. Perez, J., Fusco, G. and Moriconi-Ebrard, F. Identification and quantification of urban space in India: Defining urban macro-structures. Urban Studies, 56(10), pp. 1988-2004, (2019).

13. Mills, D.E.: Growth, speculation and sprawl in a monocentric city. Journal of Urban Economics, 10(2), pp 201-226, (1981).

14. Zang, W., Wrenn, D.H., and Irwin, E.G. Spatial Heterogeneity, Accessibility, and Zoning: An Empirical Investigation of Leapfrog Development. Journal of Economic Geography, 17(3), pp. 547-570, (2017).

\section{Supplementary Materials 1}

Files

- CV_PT_Ext_0217.gpkg $(9.548 \mathrm{kB})$

- PT_Classification_V1.R (14 Ko)

- CV_PT_Ext_0217_RES.gpkg (47.764 Ko)

Link

A Morpho-Structural Analysis to Classify Building Centroids as Patterns of Urban Growth. https://zenodo.org/record/3709131

\section{Description}

This upload contains a dataset ready to be processed, an R script and a dataset with the associated results of the processing steps. The dataset is a GeoPackage file (EPSG:2154 - RGF93 / Lambert-93 - Projected) named "CV_PT_Ext_0217.gpkg” containing two layers (1) "CV_Building_PT": a point feature class made of the centroids of residential buildings in 2002 and 2017 extracted from the French BD TOPO® (National Geographic Institute). This layer possesses two attribute columns indicative of the building presence for each period: "Pres_2002" and "Pres_2017" (modalities: "1" for building presence; "0" otherwise). (2) "Centre_Var_ext": a polygon feature class related to the extent of the case study: a region in southern France named Centre-Var. The script performs a combination of variography analysis and morphological closings over the building centroids. The different bounding regions then allow classifying new residential buildings (the ones that appeared between 2002 and 2017) into different categories of patterns of urban growth according to their degrees of clustering/scattering and to their locations regarding existing urban areas. The GeoPackage associated with the results "CV_PT_Ext_0217_RES.gpkg" contains two additional layers (3) "PT_CLASS" a point feature class made of the new building centroids with an attribute column "cat" related to the classification outputs. The categories are as follows: 1 - clustered infill, 2 - scattered infill, 3 - clustered edgeexpansion, 4 - scattered edge-expansion, 5 - clustered leapfrog and 6 - scattered leapfrog. (4) "BR_Clipping": a polygon feature class made of the different bounding regions clipped together. 